\title{
Comparative assessment of coated and uncoated ceramic tools on cutting force components and tool wear in hard turning of AISI H11 steel using Taguchi plan and RMS
}

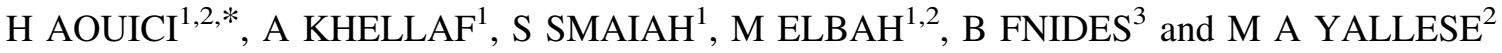 \\ ${ }^{1}$ Ecole Nationale Supérieure de Technologie, Alger, Algeria \\ ${ }^{2}$ Laboratoire Mécanique et Structure (LMS), Département de Génie Mécanique, Université 08 Mai 1945, \\ BP 401, 24000 Guelma, Algeria \\ ${ }^{3}$ Département de Construction Mécanique et Productique (CMP), FGM\&GP, Université des Sciences et de la \\ Technologie Houari Boumediene (USTHB), BP 32, El-Alia, Bab-Ezzouar, 16111 Alger, Algeria \\ e-mail: aouici_hamdi@yahoo.fr; haouici@enst.dz
}

MS received 23 May 2015; revised 4 March 2016; accepted 21 May 2017; published online 20 November 2017

\begin{abstract}
This study investigated the cutting performance of coated CC6050 and uncoated CC650 mixed ceramics in hard turning of hardened steel. The cutting performance was mainly evaluated by cutting force components and tool wear. The planning of experiments was based on Taguchi's $\mathrm{L}_{36}$ orthogonal array. The response surface methodology and analysis of variance were used to check the validity of multiple linear regression models and to determine the significant parameter affecting the cutting force components. Tool wear progressions and, hence, tool life, different tool wear forms and wear mechanisms observed for tools coated with TiN and uncoated mixed ceramics are presented along with the images captured by digital and electron microscope. Experimental observations indicate higher tool life with uncoated ceramic tools, which shows encouraging potential of these tools to hard turning of AISI H11 (50 HRC). Finally, tool performance indices are based on units which characterise machined cutting force components and wear when hard turning.
\end{abstract}

Keywords. Ceramic; ANOVA; tool wear; hard turning; RMS.

\section{Introduction}

Ceramics in recent years have been sought in many applications due to their improved properties like good thermal shock resistance, good high-temperature strength, creep resistance, low density, high hardness and wear resistance, electrical resistivity, and better chemical resistance. On the negative side, they feature low ductility and fracture toughness at the room temperature and standard pressure so that the fracture will occur once the atomic linkage forces are exceeded [1-5].

Many studies have been conducted to investigate performance of ceramic tool in the cutting of various hardened materials. Quiza et al [6] investigated hard turning of D2 steel (60 HRC) using ceramic insert $\left(70 \% \mathrm{Al}_{2} \mathrm{O}_{3}, 30 \%\right.$ TiC). It was found that, for every combination of feed rate and cutting speed, wear grows with time and significant influence on the tool wear. Lima et al [7] investigated the machinability of AISI D2 cold work tool steel (50 HRC) and AISI 4340 steel (42 HRC) using ceramic and coated carbide inserts. They observed principal wear mechanism as abrasion and

*For correspondence diffusion while machining 42 and $50 \mathrm{HRC}$ steel, respectively. In another study, Davim and Figueira [8] compared the performance of wiper and conventional ceramic cutting tool in turning D2 steel (60 HRC). Cutting time and cutting velocity were the main parameters that affect the flank wear of ceramic cutting tools. The specific cutting pressures of ceramic tools are strongly influenced by the feed rate. With wiper ceramic inserts, machined surface roughness less than $0.8 \mu \mathrm{m}$ was achieved. Recently, Elbah et al [9] compared the obtained values of surface roughness with wiper and conventional ceramic inserts during hard turning of AISI 4140 steel. They disclosed that the improved surface quality is achieved with wiper geometry. The same was reported by Gaitonde et al [10] in hard turning of AISI D2 cold work tool steel with conventional and wiper ceramic inserts.

On the other hand, in hard turning, there are various factors that affect the cutting force and the tool wear, for example, tool variables (nose radius, cutting edge geometry, rake angle, tool point angle, tool materials, tool overhang, etc.), work piece variables (material and hardness) and cutting conditions (cutting speed, feed rate and depth of cut). Successful implementation of hard turning is essential to select most suitable machining conditions to appreciate cutting 
efficiency and develop high-quality machined parts with minimum processing cost. The techniques used for optimising process parameters by means of experimental procedures and mathematical (statistical) models have increased considerably with time to accomplish a general objective of enhancing productivity and advancing cutting process efficiency. In this case, the Response surface methodology (RSM) is practical, economical and relatively easy to use, that is, many researchers have used response surface methodology [3, 11-13]. In this methodology, the effect of cutting parameters on machining outputs are obtained using a set of experiments capable of generating an appropriate dataset for efficient statistical analysis, which in turn produces valid and objective models. These models can be used in optimisation, simulation or prediction of turning process behaviour, mainly within the experimental range [14].

Horng et al [2] developed an RSM model using Central Composite Design (CCD) in the hard turning using uncoated $\mathrm{Al}_{2} \mathrm{O}_{3} / \mathrm{TiC}$ mixed ceramic tools for flank wear and surface roughness. Flank wear was influenced principally by the cutting speed and the interaction effect of feed rate with the nose radius of tool. The cutting speed and the tool corner radius affected surface roughness significantly. In another study, Bouacha et al [15] used RSM to build quadratic models for surface roughness and cutting forces in the study of AISI 52100 hardened bearing steel. After the modeling task, desirability function was used as a multi-response optimisation method. Benga and Abrão [16] have studied tool life and the surface finishing of hardened 100Cr6 bearing steel obtained with Polycrystalline Cubic Boron Nitride (PCBN) and ceramic inserts using RSM. Sahin and Motorcu [17] used RSM to model surface roughness $\left(R a, R z\right.$ and $\left.R_{\max }\right)$ in the turning of AISI 1050 hardened steel by cubic boron nitride (CBN) cutting tools. Al-Ahmari [18] built empirical models for tool life, surface roughness and cutting force in a hard turning of austenitic AISI 302.

Recently, Aouici et al [19] developed a mathematical model to study the effect of cutting parameters on the surface roughness, cutting force, cutting pressure and cutting power using the RSM. After the regression analysis and the variance analysis, it was found that the model was adequate and all the main cutting parameters had a significant impact on the cutting force, cutting pressure and cutting power. In another recent work, Meddour et al [20] applied the RSM to investigate the effect of cutting parameters on cutting forces and surface roughness in hard turning of AISI 52100 steel with a ceramic tool. The study indicated that the depth of cut is the main parameter affecting the force components, followed by feed rate.

\section{Experimental conditions and procedures}

In the turning experiments, AISI H11 hot work tool steel bars with dimensions of $\varnothing 75 \times 400 \mathrm{~mm}^{2}$ were used, which is widely used in hot form forging. It is used to manufacture module matrices of car doors, helicopter rotor blades, shells, module and inserts of high-pressure die casting strongly requested with high lifespan [21]. Its chemical composition (in wt.\%) is given as follows: $\mathrm{C} 0.35$; $\mathrm{Cr} 5.26$; Mo 1.19; V 0.50; Si 1.01; Mn 0.32; S 0.002; P 0.016; Fe 90.31 and other components 1.042. The hardness was raised by quenching and tempering treatment, followed by checking measurement with a digital Micron Hardness Tester DM2-D390. The average of measured values was 50 HRC.

The lathe, used for machining operations, was from TOS TRENCIN company; model SN40C, spindle power $6.6 \mathrm{~kW}$ and a maximum spindle speed of $2000 \mathrm{rpm}$. The cutting conditions for finish hard turning under higher parametric condition are shown in table 1 .

A tool holder and insert geometry, having the ISO designation: PSBNR2525K12 and SNGA120408T01020, respectively, were employed with tool geometry as follows: $\chi=75^{\circ} ; \alpha=6^{\circ} ; \gamma=-6^{\circ} ; \lambda=-6^{\circ}$. The three components of the cutting force - feed force $(F a)$, radial force $(F r)$ and tangential force $(F t)$ - were recorded using a standard quartz dynamometer (Kistler 9257B) allowing measurements from -5 to $5 \mathrm{kN}$. Tool flank wear was inspected several times during the tool life, using an optical microscope (Visual Machine 250). Tool life was considered ended when the flank wear reached $V B=0.30 \mathrm{~mm}$. At the end of the tool life, worn inserts were examined in a scanning electron microscope (SEM) with an embedded energy-dispersive X-ray (EDS) analyzer.

Table 1. Cutting conditions.

\begin{tabular}{lc}
\hline Cutting conditions & Descriptions \\
\hline Work piece & AISI H11 (X38CrMoV5-1) \\
Hardness & $50 \mathrm{HRC}$ \\
Cutting & Dry \\
$\quad$ environment & CC6050 coated with TiN \\
Cutting tools & CC650 conventional \\
& SNGA 120408 T01020 \\
Tool geometry & PSBNR $2525 \mathrm{~K} 12$ \\
Tool holder & $100 ; 150 ; 200$ \\
Multi-factorial method (cutting force components) \\
Cutting speed & $0.08 ; 0.14 ; 0.20$ \\
Feed & $0.1 ; 0.3 ; 0.5$ \\
Depth of cut & $0.8 ; 1.2$ \\
Cutting radius & force \\
Responses & Feed force, radial force and tangential \\
& $4 \mathrm{~min}$ \\
Single-factor method (wears) & $150 \mathrm{~m} / \mathrm{min}$ \\
Cutting time/test & $0.08 \mathrm{~mm} / \mathrm{rev}$ \\
Cutting speed & $0.30 \mathrm{~mm}$ \\
Feed & Flank and crater wears \\
Depth of cut & \\
Responses &
\end{tabular}




\section{Design of experiment}

\subsection{Orthogonal array}

In this study, a factorial design was used to identify the main effects of four factors (cutting parameters) on three responses, namely axial force $(F a)$, radial force $(F r)$ and tangential force $(F v)$ for both ceramic tools; uncoated $\mathrm{Al}_{2} \mathrm{O}_{3} / \mathrm{TiC}$ mixed (CC650) and coated $\mathrm{Al}_{2} \mathrm{O}_{3} / \mathrm{TiC}$ mixed (CC6050). The fractional factorial design selected was an $\mathrm{L}_{36}$ orthogonal array, with factors ("A," "B," "C" and "D") and three levels for ("B," "C," "D") and two levels for "A." In the matrix shown in table 3, the three levels are represented by " -1, " " 0 " and " +1, , where " -1 " is the lowest level and " +1 " is the highest one. For each experiment, 36 machining trials were carried out. The factors considered were tool nose radius $[\mathrm{mm}]$ (Factor "A"), cutting speed $[\mathrm{m} / \mathrm{min}]$ (Factor "B"), feed rate $[\mathrm{mm} /$ rev] (Factor "C") and depth of cut [mm] (Factor "D"). Their levels were chosen according to the cutting tool specifications (table 1).

\subsection{Response surface methodology}

Response surface methodology is a collection of mathematical and statistical techniques that are useful for the modelling and analysis of problems in which a response of interest is influenced by several variables and the purpose is to optimise this response [22]. RSM comprises the following three major components: (i) experimental design to determine the process factors' values based on which the experiments are conducted and data are collected; (ii) empirical modelling to approximate the relationship (i.e. the response surface) between responses and factors; (iii) optimisation to find the best response value based on the empirical model. These models can be used in optimisation, simulation or prediction of turning process behaviour, mainly within the experimental range [9].

In our study, cutting radius $(r, \mathrm{~mm})$, cutting speed $(V c, \mathrm{~m} / \mathrm{min})$, feed rate $(f, \mathrm{~mm} / \mathrm{rev})$ and depth of cut ( $a p$, $\mathrm{mm}$ ) for two different ceramics (CC6050 and CC650) have been chosen as process parameters. The cutting force components, namely axial force $(F a)$, radial force $(F r)$ and tangential force $(F t)$ of the job, have been chosen as responses factor. The relationship between the input parameters and the output parameters is given as follows:

$$
Y=\varphi(A, B, C, D)
$$

where $Y$ is the desired machinability aspect and $\varphi$ is the response function. The approximation of $Y$ is proposed by using a multiple linear mathematical model, which is suitable for studying the interaction effects of process parameters on machinability characteristics. In the present work, the RMS based on multiple linear mathematical models is given by the following equation:

$$
Y=a_{0}+\sum_{i=1}^{k} b_{i} X_{i}+\sum_{i, j}^{k} b_{i j} X_{i} X_{j}
$$

where $b_{0}$ is the free term of the regression equation, the coefficients $b_{1}, b_{2}, \ldots, b_{K}$ and $b_{12}, b_{13}, \ldots, b_{k-1}$ are the interacting terms. $X i$ represents input parameters (A, B, C and D). The output $(F a, F r$ and $F t)$ is also called the response factors. The experimental plan and result of the trials are reported in table 2. Based on Taguchi plan $1^{2} \times 3^{3}$ full factorial design, 36 tests were carried out.

\subsection{Analysis of variance}

Analysis of variance (ANOVA) can be useful to determine the influence of any given input parameters from a series of experimental results by design of experiments for machining process and it can be used to interpret experimental data. The obtained results are analyzed using Design-Expert V8, statistical analysis software that is widely used in many engineering applications. The ANOVA table consists of sum of squares and freedom degrees. The mean square is the ratio of sum of squares to freedom degrees and $F$-value is the ratio of mean square to the mean square of the experimental error. The statistical significance and the adequacy of the model have been checked using an ANOVA depending on $F$-value and $P$-value. It is commonly used to summarise the test of the regression model, test of significance factors and their interactions. If the model's $P$-value is less than 0.05 ( $95 \%$ confidence level), the significance of corresponding term is established and the model has a significant effect on the response [19]. In general, $R^{2}$ measures the percentage of data variation that is explained by the regression equation. The adjusted $R^{2}$ value is particularly useful when comparing models with different number of terms. When $R^{2}$ approaches to unity, the response model fits the actual data effectively.

\section{Results and discussion}

\subsection{Statistical analysis}

Tables 3-5 show ANOVA results, respectively, for $\mathrm{Fa}$, $\mathrm{Fr}$ and $F t$ for both ceramic tools CC650 and CC6050. This analysis was out for a $5 \%$ significance level, that is, for a 95\% confidence level. In addition to freedom degree, mean of squares (MS), sum of squares (SS), $F$-value and probability (Prob.) associated with each factor level were presented. The last column of tables shows the factor contribution (percentage; Cont. \%) on the total variation, indicating the degree of influence on the result. 
Table 2. Orthogonal table $\mathrm{L}_{36}$ for responses.

\begin{tabular}{|c|c|c|c|c|c|c|c|c|c|c|}
\hline \multirow[b]{3}{*}{ Test no. } & \multirow{2}{*}{\multicolumn{4}{|c|}{ Machining parameters }} & \multicolumn{6}{|c|}{ Cutting force components } \\
\hline & & & & & \multicolumn{3}{|c|}{ CC6050 } & \multicolumn{3}{|c|}{ CC650 } \\
\hline & $r(\mathrm{~mm})$ & $V c(\mathrm{~m} / \mathrm{min})$ & $f(\mathrm{~mm} / \mathrm{rev})$ & $a p(\mathrm{~mm})$ & $F a(\mathrm{~N})$ & $\operatorname{Fr}(\mathrm{N})$ & $F t(\mathrm{~N})$ & $F a(\mathrm{~N})$ & $F r(\mathrm{~N})$ & $F t(\mathrm{~N})$ \\
\hline 1 & 0.8 & 100 & 0.08 & 0.1 & 83.63 & 195.63 & 164.26 & 14.99 & 38.54 & 20.41 \\
\hline 2 & 0.8 & 150 & 0.14 & 0.3 & 40.27 & 115.76 & 67.30 & 19.45 & 97.38 & 78.08 \\
\hline 3 & 0.8 & 200 & 0.20 & 0.5 & 75.59 & 227.98 & 170.16 & 44.42 & 136.85 & 161.84 \\
\hline 4 & 0.8 & 100 & 0.08 & 0.1 & 45.30 & 157.66 & 72.93 & 11.80 & 44.81 & 22.71 \\
\hline 5 & 0.8 & 150 & 0.14 & 0.3 & 41.52 & 138.91 & 91.83 & 33.03 & 84.81 & 87.24 \\
\hline 6 & 0.8 & 200 & 0.20 & 0.5 & 36.47 & 150.90 & 107.03 & 53.30 & 134.61 & 153.24 \\
\hline 7 & 0.8 & 100 & 0.08 & 0.3 & 74.42 & 199.15 & 175.80 & 19.12 & 65.03 & 48.98 \\
\hline 8 & 0.8 & 150 & 0.14 & 0.5 & 60.76 & 184.56 & 120.97 & 47.19 & 126.85 & 131.83 \\
\hline 9 & 0.8 & 200 & 0.20 & 0.1 & 06.06 & 89.60 & 46.54 & 07.45 & 58.82 & 30.80 \\
\hline 10 & 0.8 & 100 & 0.08 & 0.5 & 10.70 & 74.20 & 31.40 & 47.71 & 103.29 & 100.70 \\
\hline 11 & 0.8 & 150 & 0.14 & 0.1 & 70.67 & 220.13 & 142.30 & 09.31 & 38.08 & 33.81 \\
\hline 12 & 0.8 & 200 & 0.20 & 0.3 & 70.21 & 195.13 & 104.85 & 30.35 & 111.61 & 100.96 \\
\hline 13 & 0.8 & 100 & 0.14 & 0.5 & 75.14 & 196.98 & 167.99 & 53.88 & 125.01 & 122.15 \\
\hline 14 & 0.8 & 150 & 0.20 & 0.1 & 71.93 & 175.70 & 139.75 & 09.66 & 57.89 & 31.31 \\
\hline 15 & 0.8 & 200 & 0.08 & 0.3 & 41.00 & 165.51 & 112.13 & 18.99 & 77.12 & 59.59 \\
\hline 16 & 0.8 & 100 & 0.14 & 0.5 & 07.99 & 79.15 & 41.07 & 54.63 & 118.46 & 133.28 \\
\hline 17 & 0.8 & 150 & 0.20 & 0.1 & 38.60 & 105.48 & 61.54 & 10.77 & 59.29 & 48.48 \\
\hline 18 & 0.8 & 200 & 0.08 & 0.3 & 04.88 & 51.91 & 15.35 & 22.55 & 58.81 & 51.07 \\
\hline 19 & 1.2 & 100 & 0.14 & 0.1 & 12.45 & 107.04 & 55.23 & 04.19 & 38.05 & 29.93 \\
\hline 20 & 1.2 & 150 & 0.20 & 0.3 & 13.41 & 81.48 & 25.57 & 36.71 & 122.73 & 105.89 \\
\hline 21 & 1.2 & 200 & 0.08 & 0.5 & 09.59 & 57.63 & 29.63 & 41.20 & 112.42 & 85.31 \\
\hline 22 & 1.2 & 100 & 0.14 & 0.3 & 52.46 & 202.50 & 114.91 & 27.10 & 106.18 & 87.19 \\
\hline 23 & 1.2 & 150 & 0.20 & 0.5 & 93.08 & 312.74 & 207.45 & 55.97 & 170.88 & 163.39 \\
\hline 24 & 1.2 & 200 & 0.08 & 0.1 & 28.42 & 93.20 & 63.19 & 14.22 & 38.87 & 29.48 \\
\hline 25 & 1.2 & 100 & 0.20 & 0.3 & 30.86 & 133.48 & 88.88 & 31.03 & 129.96 & 125.40 \\
\hline 26 & 1.2 & 150 & 0.08 & 0.5 & 06.77 & 56.42 & 36.19 & 49.66 & 122.11 & 90.16 \\
\hline 27 & 1.2 & 200 & 0.14 & 0.1 & 16.90 & 125.82 & 54.71 & 16.78 & 6268 & 45.67 \\
\hline 28 & 1.2 & 100 & 0.20 & 0.3 & 64.60 & 151.03 & 105.73 & 24.60 & 119.03 & 120.7 \\
\hline 29 & 1.2 & 150 & 0.08 & 0.5 & 53.02 & 240.04 & 134.58 & 44.03 & 107.31 & 96.21 \\
\hline 30 & 1.2 & 200 & 0.14 & 0.1 & 75.29 & 197.91 & 140.62 & 04.73 & 48.63 & 30.41 \\
\hline 31 & 1.2 & 100 & 0.20 & 0.5 & 18.03 & 71.84 & 48.56 & 70.23 & 179.16 & 215.75 \\
\hline 32 & 1.2 & 150 & 0.08 & 0.1 & 30.73 & 123.79 & 45.98 & 06.44 & 28.04 & 14.49 \\
\hline 33 & 1.2 & 200 & 0.14 & 0.3 & 17.78 & 112.10 & 64.73 & 26.69 & 100.03 & 70.91 \\
\hline 34 & 1.2 & 100 & 0.20 & 0.1 & 49.63 & 217.59 & 123.94 & 08.35 & 60.08 & 51.11 \\
\hline 35 & 1.2 & 150 & 0.08 & 0.3 & 73.22 & 204.60 & 106.50 & 23.59 & 79.76 & 43.12 \\
\hline 36 & 1.2 & 200 & 0.14 & 0.5 & 56.08 & 229.30 & 134.34 & 41.40 & 142.87 & 139.49 \\
\hline
\end{tabular}

Table 3 shows the results of ANOVA for feed force of coated ceramic (CC6050) and uncoated ceramic (CC650) tools. From the analysis of table 3, it can be apparent seen that the model is significant and the depth of cut is the most important factor affecting $F a$. Its contribution is (95.03 and 91.68)\%. This is because increased depth of cut results in increased tool work contact length [23]. Subsequently, chip thickness becomes significant that causes the volume growth of deformed metal, requiring greater cutting forces to cut the chip. However, a qualitative comparison can be made; for example, Aouici et al [22] found that the depth of cut and feed rate are the important factors affecting $F t$ when the hard turning of AISI H11 (50HRC) with CBN7020 tool. The next factor influencing $\mathrm{Fa}$ is the feed rate with (1 and 2.07)\% contribution, which has a very weak significance effect, for CC650 and CC6050 tools, respectively.

The other important coefficient $R^{2}$ in the resulting ANOVA table is defined as the ratio of the explained variation to the total variation, and it is a measure of the degree of fit. When $R^{2}$ approaches to unity, the better response model fits the actual data. The value of $R^{2}$ calculated in table 3 for these models are over 0.94 for both ceramic tools CC6050 and CC650, respectively, and reasonably close to unity, which is acceptable. It denotes that about $95 \%$ of the variability in the data is explained by 
Table 3. ANOVA result for axial force $(F a)$.

\begin{tabular}{|c|c|c|c|c|c|c|c|}
\hline Source & SS & $\mathrm{DF}$ & MS & F-value & Prob. & Cont. \% & Remarks \\
\hline \multicolumn{8}{|l|}{ (a) $C C 6050$} \\
\hline Model & 23871.442 & 10 & 2387.1442 & 84.10835928 & $<0.0001$ & & Significant \\
\hline A-r, $\mathrm{mm}$ & 152.59279 & 1 & 152.59279 & 5.376436573 & 0.0289 & 0.65 & Significant \\
\hline $\mathrm{B}-V c, \mathrm{~m} / \mathrm{min}$ & 539.32575 & 1 & 539.3257 & 19.00253991 & 0.0002 & 2.29 & Significant \\
\hline $\mathrm{C}-f, \mathrm{~mm} / \mathrm{rev}$ & 236.11782 & 1 & 236.11782 & 8.319347538 & 0.0080 & 1.00 & Significant \\
\hline $\mathrm{D}-a p, \mathrm{~mm}$ & 22402.315 & 1 & 22402.315 & 789.3205271 & $<0.0001$ & 95.03 & Significant \\
\hline $\mathrm{AB}$ & 63.773986 & 1 & 63.773986 & 2.247005128 & 0.1464 & 0.27 & Insignificant \\
\hline $\mathrm{AC}$ & 93.487746 & 1 & 93.487746 & 3.293936214 & 0.0816 & 0.40 & Insignificant \\
\hline $\mathrm{AD}$ & 0.5356070 & 1 & 0.5356070 & 0.018871517 & 0.8918 & 0.00 & Insignificant \\
\hline $\mathrm{BC}$ & 3.5628846 & 1 & 3.5628846 & 0.125534257 & 0.7261 & 0.02 & Insignificant \\
\hline $\mathrm{BD}$ & 31.069443 & 1 & 31.069443 & 1.094697107 & 0.3054 & 0.13 & Insignificant \\
\hline $\mathrm{CD}$ & 52.060370 & 1 & 52.060370 & 1.834288964 & 0.1877 & 0.22 & Insignificant \\
\hline Residual & 709.54429 & 25 & 28.381771 & & & & \\
\hline Lack of fit & 529.50249 & 16 & 33.093905 & 1.654311132 & 0.2241 & & Significant \\
\hline Pure error & 180.0418 & 9 & 20.004644 & & & & \\
\hline Cor total & 24580.986 & 35 & & & \multicolumn{3}{|c|}{100} \\
\hline \multicolumn{5}{|l|}{$\mathrm{SD}=5.33$} & \multicolumn{3}{|c|}{$R^{2}=0.9711$} \\
\hline \multicolumn{5}{|l|}{ Mean $=43.26$} & \multicolumn{3}{|c|}{$R^{2}$ adjusted $=0.9596$} \\
\hline \multicolumn{5}{|c|}{ Coefficient of variation $=12.31$} & \multicolumn{3}{|c|}{$R^{2}$ predicted $=0.9338$} \\
\hline \multicolumn{5}{|c|}{$\begin{array}{l}\text { Predicted residual error of sum of squares (PRESS) }=1627.48 \\
\text { (b) } C C 650\end{array}$} & \multicolumn{3}{|c|}{ Adequate precision $=27.796$} \\
\hline Model & 10580.801 & 10 & 1058.0801 & 43.146094 & $<0.0001$ & & Significant \\
\hline $\mathrm{A}-r, \mathrm{~mm}$ & 2.5028877 & 1 & 2.5028877 & 0.1020620 & 0.7520 & 0.02 & Insignificant \\
\hline $\mathrm{B}-V c, \mathrm{~m} / \mathrm{min}$ & 94.419445 & 1 & 94.419445 & 3.8502094 & 0.0610 & 0.88 & Insignificant \\
\hline $\mathrm{C}-f, \mathrm{~mm} / \mathrm{rev}$ & 221.04473 & 1 & 221.04473 & 9.0136994 & 0.0060 & 2.07 & Significant \\
\hline $\mathrm{D}-a p, \mathrm{~mm}$ & 9798.2127 & 1 & 9798.2127 & 399.54874 & $<0.0001$ & 91.68 & Significant \\
\hline $\mathrm{AB}$ & 32.362811 & 1 & 32.362811 & 1.3196815 & 0.2615 & 0.30 & Insignificant \\
\hline $\mathrm{AC}$ & 12.068887 & 1 & 12.068887 & 0.4921416 & 0.4895 & 0.11 & Insignificant \\
\hline $\mathrm{AD}$ & 70.509675 & 1 & 70.509675 & 2.8752235 & 0.1024 & 0.66 & Insignificant \\
\hline $\mathrm{BC}$ & 3.1093525 & 1 & 3.1093525 & 0.1267922 & 0.7248 & 0.03 & Insignificant \\
\hline $\mathrm{BD}$ & 183.00331 & 1 & 183.00331 & 7.4624573 & 0.0114 & 1.71 & Significant \\
\hline $\mathrm{CD}$ & 270.21561 & 1 & 270.21561 & 11.018776 & 0.0028 & 2.53 & Significant \\
\hline Residual & 613.07993 & 25 & 24.523197 & & & & \\
\hline Lack of fit & 360.00023 & 16 & 22.500014 & 0.8001437 & 0.6663 & & Insignificant \\
\hline Pure error & 253.0797 & 9 & 28.119966 & & & & \\
\hline Cor total & 11193.881 & 35 & & & \multicolumn{3}{|c|}{100} \\
\hline \multicolumn{5}{|l|}{$\mathrm{SD}=4.95$} & \\
\hline \multicolumn{5}{|l|}{ Mean $=28.76$} & \multicolumn{3}{|c|}{$R^{2}$ adjusted $=0.9233$} \\
\hline \multicolumn{5}{|c|}{ Coefficient of variation $=17.22$} & \multicolumn{3}{|c|}{$R^{2}$ predicted $=0.8843$} \\
\hline \multicolumn{5}{|c|}{ Predicted residual error of sum of squares $($ PRESS $)=1294.61$} & \multicolumn{3}{|c|}{ Adequate precision $=22.070$} \\
\hline
\end{tabular}

these models. It also confirms that these models provide an excellent explanation of the relationship between the independent factors and the response.

ANOVA table for response surface quadratic model for radial force $\mathrm{Fr}$ using two ceramic tools (CC6050 and CC650) is shown in table 4. The factors, depth of cut and feed rate, are significant as their $P$-value is less than 0.05 . From table 4 , it can be seen that the most effective variable on the $F r$ value is the depth of cut. Its contribution is (73.84 and 81.48$) \%$. The other variables that have effect on $\mathrm{Fr}$ are feed rate and tool nose radius with [(11.28 and 12.60) and (8.57 and 2.11)]\% for CC6050 and CC650 tools, respectively.
The $R^{2}$ value is high, close to 1 , which is desirable. The " $R$-Squared" of (0.9562 and 0.9768) are in reasonable agreement with the "Adj $R$-Squared" of (0.9386 and 0.9676) for CC6050 and CC650 tools, respectively.

Regarding tangential force, as shown in table 5, the percentage contributions of factors $\mathrm{A}, \mathrm{B}, \mathrm{C}$ and D on the $F t$ for both ceramic tools CC6050 and CC650 are [(0.23 and $0.10)$, (0.38 and 0.28), (15.62 and 15.65) and (81.56 and 78.06)]\% respectively. In this case, the most effective parameter for the tangential force is factor D; namely, the depth of cut, because increasing depth of cut increases the chip volume removed. The next largest factor influencing $F t$ is feed rate (C) with (15.62 and 15.65)\% for CC650 and 
Table 4. ANOVA result for radial force $(F r)$.

\begin{tabular}{|c|c|c|c|c|c|c|c|}
\hline Source & SS & DF & MS & F-value & Prob. & Cont. \% & Remarks \\
\hline \multicolumn{8}{|l|}{ (a) $C C 6050$} \\
\hline Model & 132702.01 & 10 & 13270.201 & 54.53223659 & $<0.0001$ & & Significant \\
\hline $\mathrm{A}-r, \mathrm{~mm}$ & 9893.0503 & 1 & 9893.0503 & 40.65425666 & $<0.0001$ & 8.57 & Significant \\
\hline $\mathrm{B}-V c, \mathrm{~m} / \mathrm{min}$ & 4652.4128 & 1 & 4652.4128 & 19.1185103 & 0.0002 & 4.03 & Significant \\
\hline $\mathrm{C}-f, \mathrm{~mm} / \mathrm{rev}$ & 13027.091 & 1 & 13027.091 & 53.53320945 & $<0.0001$ & 11.28 & Significant \\
\hline D-ap, mm & 85270.913 & 1 & 85270.913 & 350.4101838 & $<0.0001$ & 73.84 & Significant \\
\hline $\mathrm{AB}$ & 259.24390 & 1 & 259.24390 & 1.065330482 & 0.3119 & 0.22 & Insignificant \\
\hline $\mathrm{AC}$ & 142.50831 & 1 & 142.50831 & 0.58562013 & 0.4513 & 0.12 & Insignificant \\
\hline $\mathrm{AD}$ & 836.13792 & 1 & 836.13792 & 3.436004542 & 0.0756 & 0.72 & Insignificant \\
\hline $\mathrm{BC}$ & 727.19028 & 1 & 727.19028 & 2.988297778 & 0.0962 & 0.63 & Insignificant \\
\hline $\mathrm{BD}$ & 121.46520 & 1 & 121.46520 & 0.499146116 & 0.4864 & 0.11 & Insignificant \\
\hline $\mathrm{CD}$ & 555.18290 & 1 & 555.18290 & 2.281454921 & 0.1435 & 0.48 & Insignificant \\
\hline Residual & 6083.6497 & 25 & 243.34598 & & & & \\
\hline Lack of fit & 5802.2779 & 16 & 362.64237 & 11.59953253 & 0.0004 & & Significant \\
\hline Pure error & 281.3718 & 9 & 31.263533 & & & & \\
\hline Cor total & 138785.66 & 35 & & & \multicolumn{3}{|c|}{100} \\
\hline \multicolumn{5}{|l|}{$\mathrm{SD}=15.60$} & \multicolumn{3}{|c|}{$R^{2}=0.9562$} \\
\hline \multicolumn{5}{|l|}{ Mean $=151.19$} & \multicolumn{3}{|c|}{$R^{2}$ adjusted $=0.9386$} \\
\hline \multicolumn{5}{|c|}{ Coefficient of variation $=10.32$} & \multicolumn{3}{|c|}{$R^{2}$ predicted $=0.9021$} \\
\hline \multicolumn{5}{|c|}{$\begin{array}{l}\text { Predicted residual error of sum of squares }(\text { PRESS })=13587.39 \\
\text { (b) } C C 650\end{array}$} & \multicolumn{3}{|c|}{ Adequate precision $=28.535$} \\
\hline Model & 54586.456 & 10 & 5458.645649 & 105.51335 & $<0.0001$ & & Significant \\
\hline $\mathrm{A}-r, \mathrm{~mm}$ & 1092.4548 & 1 & 1092.45483 & 21.116698 & 0.0001 & 2.11 & Significant \\
\hline $\mathrm{B}-V c, \mathrm{~m} / \mathrm{min}$ & 0.9683771 & 1 & 0.968377103 & 0.0187183 & 0.8923 & 0.01 & Insignificant \\
\hline $\mathrm{C}-f, \mathrm{~mm} / \mathrm{rev}$ & 6518.7769 & 1 & 6518.776948 & 126.00525 & $<0.0001$ & 12.60 & Significant \\
\hline $\mathrm{D}-a p, \mathrm{~mm}$ & 42171.520 & 1 & 42171.52007 & 815.15797 & $<0.0001$ & 81.48 & Significant \\
\hline $\mathrm{AB}$ & 0.8985275 & 1 & 0.898527572 & 0.0173681 & 0.8962 & 0.01 & Insignificant \\
\hline $\mathrm{AC}$ & 261.29682 & 1 & 261.2968277 & 5.0507592 & 0.0337 & 0.50 & Significant \\
\hline $\mathrm{AD}$ & 886.20535 & 1 & 886.2053547 & 17.129981 & 0.0003 & 1.71 & Significant \\
\hline $\mathrm{BC}$ & 2.1848204 & 1 & 2.184820432 & 0.0422316 & 0.8388 & 0.01 & Insignificant \\
\hline $\mathrm{BD}$ & 225.39378 & 1 & 225.3937879 & 4.3567683 & 0.0472 & 0.44 & Significant \\
\hline $\mathrm{CD}$ & 595.70688 & 1 & 595.7068846 & 11.514766 & 0.0023 & 1.15 & Significant \\
\hline Residual & 1293.3542 & 25 & 51.73416826 & & & & \\
\hline Lack of Fit & 734.17350 & 16 & 45.88584415 & 0.7385315 & 0.7142 & & Insignificant \\
\hline Pure Error & 559.1807 & 9 & 62.13118889 & & & & \\
\hline Cor Total & 55879.810 & 35 & & & \multicolumn{3}{|c|}{100} \\
\hline \multicolumn{5}{|l|}{$\mathrm{SD}=7.1926$} & \multicolumn{3}{|c|}{$R^{2}=0.9769$} \\
\hline \multicolumn{5}{|l|}{ Mean $=91.834$} & \multicolumn{3}{|c|}{$R^{2}$ adjusted $=0.9676$} \\
\hline \multicolumn{5}{|c|}{ Coefficient of variation $=7.8321$} & \multicolumn{3}{|c|}{$R^{2}$ predicted $=0.9546$} \\
\hline \multicolumn{5}{|c|}{ Predicted residual error of sum of squares $($ PRESS $)=2536.264$} & \multicolumn{3}{|c|}{ Adequate precision $=36.999$} \\
\hline
\end{tabular}

CC6050 tools, respectively. The cutting speed and the tool nose radius do not present any statistical significance on the tangential force.

The $R^{2}$ value is high, close to 1 , which is desirable. The " $R$-Squared" of (0.9538 and 0.9750) are in reasonable agreement with the "Adj $R$-Squared" of (0.9354 and 0.9650) for CC6050 and CC650 tools, respectively.

\subsection{Mathematical modelling}

Regression is a technique for investigating functional relationship between output and input decision variables of a process and may be useful for manufacturing process data description, parameter estimation, and control [9]. The mathematical models determined by multiple linear regression analysis to predict the axial force, radial force and tangential force during hard turning of AISI H11 hot work tool steel using different ceramic inserts are given by the following:

\section{Cc6050}

$$
\begin{aligned}
F a_{C C 6050}= & -72.135+73.433 r+0.168 V c+251.146 f \\
& +156.545 a p-0.205 r \times V c-207.611 r \times f \\
& -3.940 r \times a p-0.207 V c \times f-0.152 V c \\
& \times a p+164.397 f \times a p
\end{aligned}
$$

$R^{2}=0.9711$ 
Table 5. ANOVA result for tangential force $(F t)$.

\begin{tabular}{|c|c|c|c|c|c|c|c|}
\hline Source & SS & DF & MS & F-value & Prob. & Cont. \% & Remarks \\
\hline \multicolumn{8}{|l|}{ (a) $C 66050$} \\
\hline Model & 83350.822 & 10 & 8335.0822 & 51.6559099 & $<0.0001$ & & Significant \\
\hline A-r, $\mathrm{mm}$ & 180.15543 & 1 & 180.15543 & 1.116496795 & 0.3008 & 0.23 & Insignificant \\
\hline $\mathrm{B}-V c, \mathrm{~m} / \mathrm{min}$ & 306.50024 & 1 & 306.50024 & 1.899507211 & 0.1803 & 0.38 & Insignificant \\
\hline $\mathrm{C}-f, \mathrm{~mm} / \mathrm{rev}$ & 12496.522 & 1 & 12496.522 & 77.44605472 & $<0.0001$ & 15.62 & Significant \\
\hline $\mathrm{D}-a p, \mathrm{~mm}$ & 65252.167 & 1 & 65252.167 & 404.394337 & $<0.0001$ & 81.56 & Significant \\
\hline $\mathrm{AB}$ & 134.81677 & 1 & 134.81677 & 0.835514632 & 0.3694 & 0.17 & Insignificant \\
\hline $\mathrm{AC}$ & 76.881684 & 1 & 76.881684 & 0.476467203 & 0.4964 & 0.10 & Insignificant \\
\hline $\mathrm{AD}$ & 0.0021559 & 1 & 0.0021559 & $1.33612 \mathrm{E}-05$ & 0.9971 & 0.01 & Insignificant \\
\hline $\mathrm{BC}$ & 317.61405 & 1 & 317.61403 & 1.968383923 & 0.1729 & 0.40 & Insignificant \\
\hline $\mathrm{BD}$ & 1.4316313 & 1 & 1.4316313 & 0.008872405 & 0.9257 & 0.01 & Insignificant \\
\hline $\mathrm{CD}$ & 1243.6552 & 1 & 1243.6552 & 7.707439863 & 0.0103 & 1.55 & Significant \\
\hline Residual & 4033.9441 & 25 & 161.35776 & & & & \\
\hline Lack of fit & 3554.8741 & 16 & 222.17963 & 4.173954728 & 0.0175 & & Significant \\
\hline Pure error & 479.07005 & 9 & 53.230005 & & & & \\
\hline Cor total & 87384.767 & 35 & & & \multicolumn{3}{|c|}{$\begin{array}{c}100 \\
R^{2}=0.9538\end{array}$} \\
\hline \multicolumn{5}{|l|}{$\mathrm{SD}=12.70$} & \multicolumn{3}{|c|}{$R^{2}=0.9538$} \\
\hline \multicolumn{5}{|l|}{ Mean $=94.83$} & \multicolumn{3}{|c|}{$R^{2}$ adjusted $=0.9354$} \\
\hline \multicolumn{5}{|c|}{ Coefficient of variation $=13.39$} & \multicolumn{3}{|c|}{$R^{2}$ predicted $=0.8930$} \\
\hline \multicolumn{5}{|c|}{$\begin{array}{l}\text { Predicted residual error of sum of squares }(\text { PRESS })=9346.30 \\
\text { (b) } C C 650\end{array}$} & \multicolumn{3}{|c|}{ Adequate precision $=24.024$} \\
\hline Model & 83941.653 & 10 & 8394.1653 & 97.54966654 & $<0.0001$ & & Significant \\
\hline A-r, $\mathrm{mm}$ & 77.543400 & 1 & 77.543400 & 0.90114175 & 0.3516 & 0.10 & Insignificant \\
\hline $\mathrm{B}-V c, \mathrm{~m} / \mathrm{min}$ & 213.64240 & 1 & 213.64240 & 2.482765634 & 0.1277 & 0.28 & Insignificant \\
\hline $\mathrm{C}-f, \mathrm{~mm} / \mathrm{rev}$ & 12126.858 & 1 & 12126.858 & 140.9277735 & $<0.0001$ & 15.65 & Significant \\
\hline $\mathrm{D}-a p, \mathrm{~mm}$ & 60474.936 & 1 & 60474.936 & 702.7869527 & $<0.0001$ & 78.06 & Significant \\
\hline $\mathrm{AB}$ & 7.8975154 & 1 & 7.8975154 & 0.091778035 & 0.7644 & 0.01 & Insignificant \\
\hline $\mathrm{AC}$ & 127.14514 & 1 & 127.14514 & 1.477569932 & 0.2355 & 0.16 & Insignificant \\
\hline $\mathrm{AD}$ & 135.67617 & 1 & 135.67617 & 1.576710171 & 0.2208 & 0.18 & Insignificant \\
\hline $\mathrm{BC}$ & 270.39466 & 1 & 270.39466 & 3.142290904 & 0.0885 & 0.35 & Insignificant \\
\hline $\mathrm{BD}$ & 331.22563 & 1 & 331.22563 & 3.849215434 & 0.0610 & 0.43 & Insignificant \\
\hline $\mathrm{CD}$ & 3708.6389 & 1 & 3708.6389 & 43.09856663 & $<0.0001$ & 4.79 & Significant \\
\hline Residual & 2151.2542 & 25 & 86.050169 & & & & \\
\hline Lack of fit & 1678.2582 & 16 & 104.89114 & 1.995831638 & 0.1472 & & Insignificant \\
\hline Pure error & 472.99595 & 9 & 52.555105 & & & & \\
\hline Cor total & 86092.907 & 35 & & & \multirow{2}{*}{\multicolumn{3}{|c|}{$R^{2}=0.9750$}} \\
\hline \multicolumn{5}{|l|}{$\mathrm{SD}=9.28$} & \multicolumn{2}{|c|}{$R^{2}=0.9750$} & \\
\hline \multicolumn{5}{|l|}{ Mean $=82.25$} & \multicolumn{3}{|c|}{$R^{2}$ adjusted $=0.9650$} \\
\hline \multicolumn{5}{|c|}{ Coefficient of variation $=11.28$} & & redicted $=$ & 381 \\
\hline \multicolumn{5}{|c|}{ Predicted residual error of sum of squares $($ PRESS) $=5332.55$} & \multicolumn{3}{|c|}{ Adequate precision $=34.964$} \\
\hline
\end{tabular}

$$
\begin{aligned}
F r_{C C 6050}= & -156.815+150.616 r+0.589 V c+997.843 f \\
& +112.383 a p-0.414 r \times V c-256.326 r \times f \\
& +155.690 r \times a p-2.956 V c \times f-0.301 V c \\
& \times a p+536.859 f \times a p
\end{aligned}
$$

\section{CC650}

$$
\begin{aligned}
F a_{C C 650}= & +15.675-23.529 r-0.055 V c+51.039 f \\
& +58.86 a p+0.146 r \times V c-74.594 r \times f \\
& +45.211 r \times a p-0.193 V c \times f-0.369 V c \\
& \times a p+374.539 f \times a p
\end{aligned}
$$

$\left(R^{2}=0.9452\right)$

$$
\begin{aligned}
F t_{C C 6050}= & -34.415-5.055 r-0.120 V c+689.979 f \\
& +143.563 a p+0.299 r \times V c-188.271 r \times f \\
& -0.250 r \times a p-1.953 V c \times f+0.032 V c \\
& \times a p+803.512 f \times a p
\end{aligned}
$$

$\left(R^{2}=0.9538\right)$

$$
\begin{aligned}
& F r_{C C 650}=+58.432-67.374 r+0.08 V c-213.412 f \\
&+33.025 a p+0.024 r \times V c+374.088 r \times f \\
&+160.283 r \times a p+0.162 V c \times f-0.410 V c \\
& \times a p+556.107 f \times a p \\
&\left(R^{2}=0.9769\right)
\end{aligned}
$$




$$
\begin{aligned}
F t_{C C 650}= & -13.384-33.066 r+0.403 V c+55.019 f \\
& +68.655 a p-0.072 r \times V c+242.115 r \times f \\
& +62.715 r \times a p-1.802 V c \times f-0.497 V c \\
& \times a p+1387.553 f \times a p
\end{aligned}
$$

$\left(R^{2}=0.9750\right)$

\subsection{Surface topography}

The two-factor interaction effects due to cutting speed $(V c)$-cutting radius $(r)$ and depth of cut ( $a p)$-feed rate $(f)$ on axial force $(F a)$, radial force $(F r)$ and tangential force $(F t)$ during hard turning of AISI H11 (50HRC) hot work tool steel were analyzed for two different ceramic inserts, namely CC6050 and CC650 through surface plots (figures 1-3). The three-dimensional (3D) response surface plots were generated considering two machining parameters at a time, while the other parameter was kept at the middle level.

From interaction plot figure 1a, it can be observed that, at a constant cutting radius, the axial force sharply decreases with the increase of cutting speed. This trend is mainly due to the increase in temperature at shear plane region, resulting in the plastic softening of this primary deformation zone and hence reduced shear strength of the material. This will in turn reduce the force required to deform the material to be machined [24]. On the other hand, axial force has a tendency to increase with the increase of cutting radius at a constant cutting speed. The lower value results with the combination of high cutting speed and low cutting radius for both ceramic tools (CC6050 and CC650). Figure 1b indicates that, with the feed rate from 0.08 to $0.20 \mathrm{~mm}$, the axial force is highly sensitive to depth of cut, this is because the increase in depth of cut results the increase of tool work contact length [23]. From figure $1 b$, it can be seen that the feed rate does not much influence the axial force for both ceramic tools CC6050 and CC650. A comparison shows that the CC6050-coated ceramic insert seems to be higher values as compared with conventional CC650 inserts.

Figure 2 shows the relations of cutting speed $(V c)$-cutting radius $(r)$ and depth of cut $(a p)$ - feed rate $(f)$ for both ceramic tools CC6050 and CC650. Figure 1a indicates that for a given cutting speed, the radial force increases with the increase of cutting radius, this is because increasing cutting radius results in the increase of tool work contact length [23]. On the other hand, cutting speed has less effect on radial force. As seen clearly in figure $2 b$, there is a regular relationship between the cutting parameters, depth of cut (ap)-feed rate $(f)$ and the radial force of CC6050 and CC650, that is, $F r$ values increase with the increase of depth of cut for CC6050 tool, at constant feed rate.
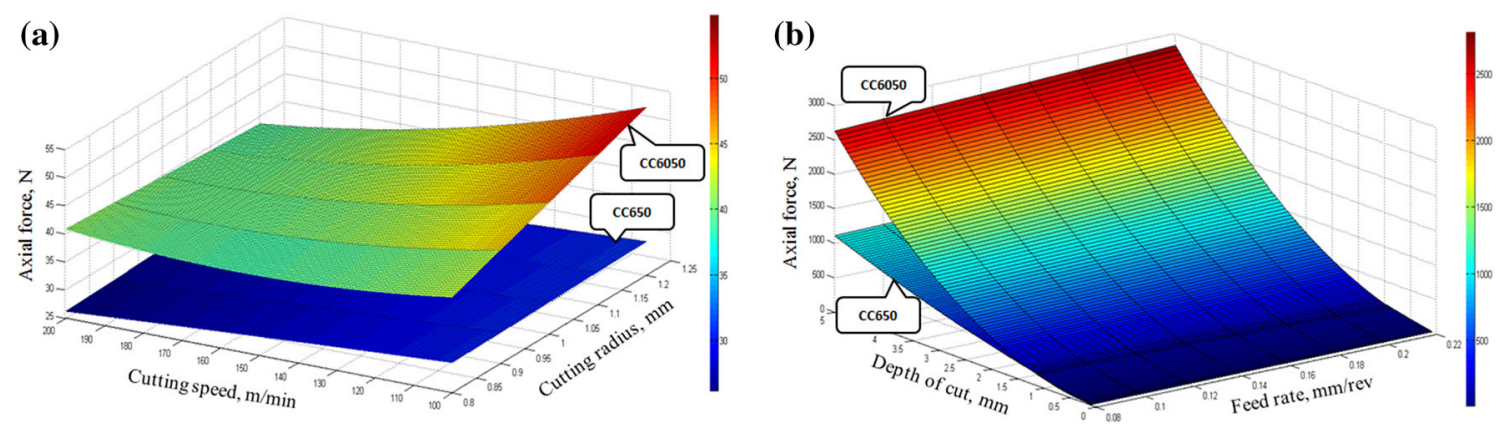

Figure 1. Three-dimensional surface plots for interaction effects of cutting speed and cutting radius (a) and (b) depth of cut and feed rate on axial force for (CC6050 and CC650).
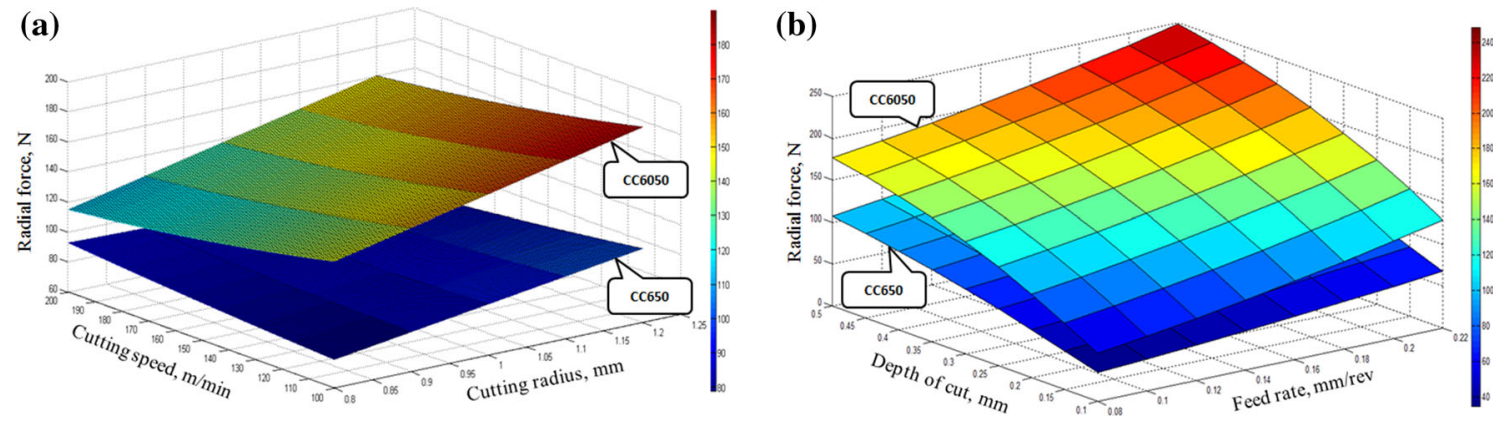

Figure 2. Three-dimensional surface plots for interaction effects of cutting speed and cutting radius (a) and (b) depth of cut and feed rate on radial force for (CC6050 and CC650). 

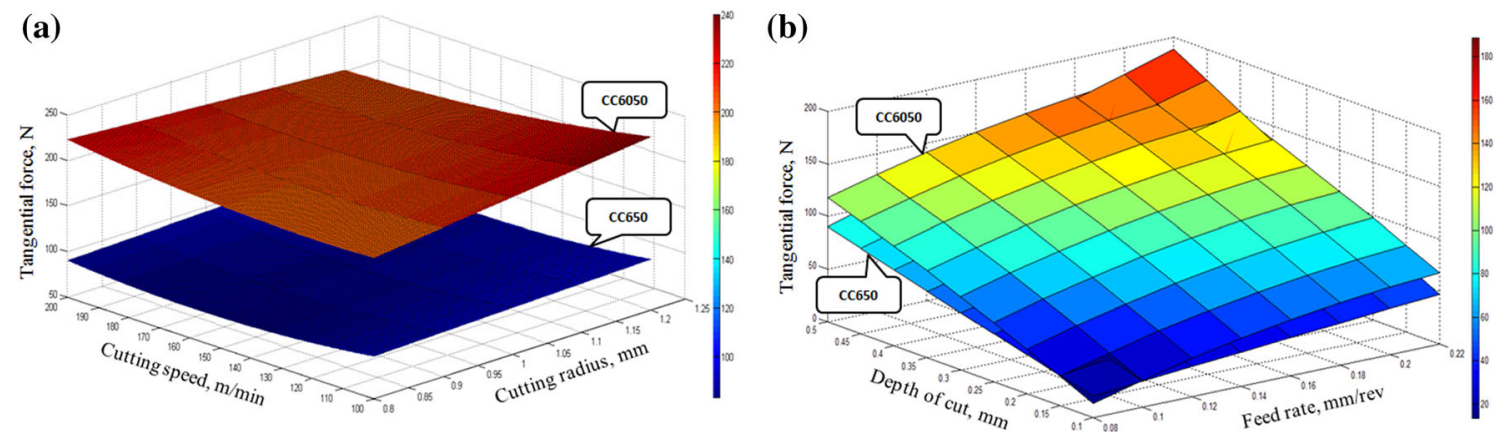

Figure 3. Three-dimensional surface plots for interaction effects of cutting speed and cutting radius (a) and (b) depth of cut and feed rate on tangential force for (CC6050 and CC650).

Table 6. Goals and parameter ranges for optimisation of cutting conditions.

\begin{tabular}{|c|c|c|c|c|c|}
\hline \multirow[b]{2}{*}{ Conditions } & \multirow[b]{2}{*}{ Goal } & \multicolumn{2}{|c|}{ Lower limit } & \multicolumn{2}{|c|}{ Upper limit } \\
\hline & & CC6050 & CC650 & CC6050 & CC650 \\
\hline Cutting radius, $r(\mathrm{~mm})$ & In range & 0.80 & & 0.12 & \\
\hline Cutting speed, $V c(\mathrm{~m} / \mathrm{min})$ & In range & 100 & & 200 & \\
\hline Feed rate, $f(\mathrm{~mm} / \mathrm{rev})$ & In range & 0.08 & & 0.20 & \\
\hline Depth of cut, ap (mm) & In range & 0.10 & & 0.50 & \\
\hline Axial force, $F a(\mathrm{~N})$ & Minimise & 4.88 & 4.19 & 93.08 & 70.23 \\
\hline Radial force, $F r(\mathrm{~N})$ & Minimise & 51.91 & 28.04 & 312.74 & 179.16 \\
\hline Tangential force, $F t(\mathrm{~N})$ & Minimise & 15.35 & 14.49 & 207.45 & 215.75 \\
\hline
\end{tabular}

Table 7. Response optimisation for cutting force components.

\begin{tabular}{|c|c|c|c|c|c|c|c|c|c|}
\hline \multirow[b]{2}{*}{ Test $\mathrm{N}^{\circ}$} & \multicolumn{4}{|c|}{ Machining parameters } & \multicolumn{3}{|c|}{ Cutting force components } & \multirow[b]{2}{*}{ Desirability } & \multirow[b]{2}{*}{ Remarks } \\
\hline & $r(\mathrm{~mm})$ & $V c(\mathrm{~m} / \mathrm{min})$ & $f(\mathrm{~mm} / \mathrm{rev})$ & ap $(\mathrm{mm})$ & $F a(\mathrm{~N})$ & $F r(\mathrm{~N})$ & $F t(\mathrm{~N})$ & & \\
\hline \multicolumn{10}{|l|}{ CC6050 } \\
\hline 1 & 0.80 & 199.99 & 0.08 & 0.10 & 4.4637 & 53.3644 & 18.7292 & 0.992 & \multirow[t]{5}{*}{ Selected } \\
\hline 2 & 0.80 & 196.60 & 0.08 & 0.10 & 4.567 & 53.4327 & 18.8777 & 0.992 & \\
\hline 3 & 0.80 & 188.96 & 0.08 & 0.10 & 4.78648 & 53.4782 & 19.1181 & 0.991 & \\
\hline 4 & 0.80 & 197.98 & 0.08 & 0.10 & 4.59515 & 53.6804 & 18.98 & 0.991 & \\
\hline 5 & 0.80 & 195.32 & 0.08 & 0.10 & 4.65002 & 53.6497 & 19.0735 & 0.991 & \\
\hline \multicolumn{10}{|l|}{ CC650 } \\
\hline 1 & 1.20 & 100.00 & 0.08 & 0.10 & 5.46516 & 29.0596 & 12.3822 & 0.991 & \multirow[t]{5}{*}{ Selected } \\
\hline 2 & 1.20 & 100.00 & 0.08 & 0.10 & 5.50183 & 29.5935 & 12.944 & 0.990 & \\
\hline 3 & 1.20 & 100.00 & 0.08 & 0.10 & 5.65692 & 29.4228 & 12.6919 & 0.990 & \\
\hline 4 & 1.20 & 100.00 & 0.08 & 0.10 & 5.37991 & 30.2136 & 13.6584 & 0.989 & \\
\hline 5 & 1.19 & 100.00 & 0.08 & 0.10 & 5.47612 & 30.0286 & 13.3972 & 0.989 & \\
\hline
\end{tabular}

Similarly, the $F r$ value obtained was high for CC650 cutting tool at a constant depth of cut. In general, the CC650 tool gives lower value results than CC6050, as reported by Aouici et al [19]. The authors documented that the feed rate and depth of cut have influenced cutting force with hard turning of AISI D3 with CC6050 tool.

The analysis of response variable can be explained through surface plots too and a typical 3D surface plot shown in figure 3a. The surface plot illustrates that cutting speed and cutting radius increase at constant feed and depth of cut, $0.14 \mathrm{~mm} / \mathrm{rev}$ and $0.30 \mathrm{~mm}$, respectively. As it can be deduced from this figure, the tangential force is not statistically significant. On the other hand, the relationship between the tangential force and both depth of cut and feed rate is plotted in figure $3 \mathrm{~b}$. As it was expected, the tangential force increases with the increase of depth of cut and 


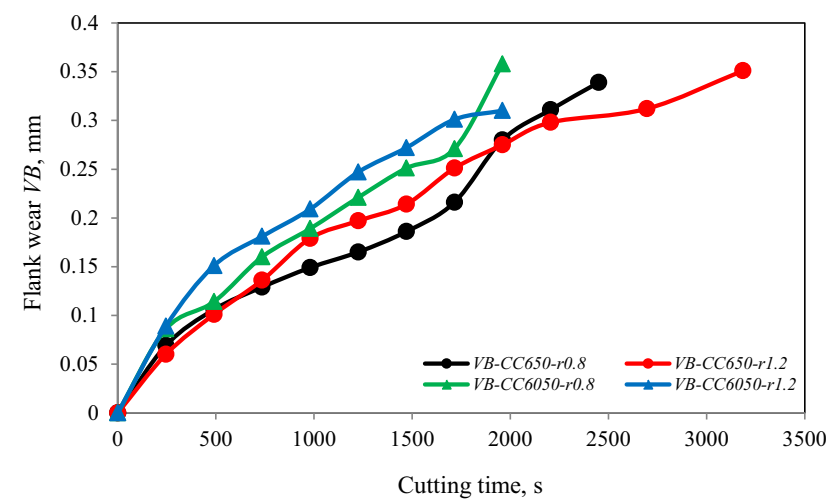

Figure 4. Flank wear evolution as a function of cutting time at various cutting radius for $V c=150 \mathrm{~m} / \mathrm{min}, f=0.08 \mathrm{~mm}$ and $a p=0.30 \mathrm{~mm}$ (CC6050 and CC650).

feed rate due to the enlargement of cutting action area [22]. Additionally, it reaches its maximum value at high levels of depth of cut and feed rate.

\subsection{Multiple response optimisations}

In the present study, desirability function optimisation of the RSM has been employed for single and multiple objective optimisations [19]. During the optimisation process, the main aim was to find out the optimal values of cutting parameters in order to minimise the cutting force components during hard turning process. Because the machining forces are the main contributing factor for power requirement, motor selection and machine tool design in machining application. These forces also affect the surface finish of the job so machinability will be good if the forces are less. The constraints used during the optimisation process are summarised in table 6 . The best (optimum) cutting conditions leading to the minimum machining forces are reported in table 7 in order to decrease the desirability level. Table 7 shows the optimisation results. Values of optimal cutting parameters are found to be as follows: $r=[0.8$ and 1.2$] \mathrm{mm}, \quad V c=[200$ and 100$] \mathrm{m} / \mathrm{min}$, $f=0.08 \mathrm{~mm} / \mathrm{rev}$ and $a p=0.10 \mathrm{~mm}$ when using uncoated

(a)

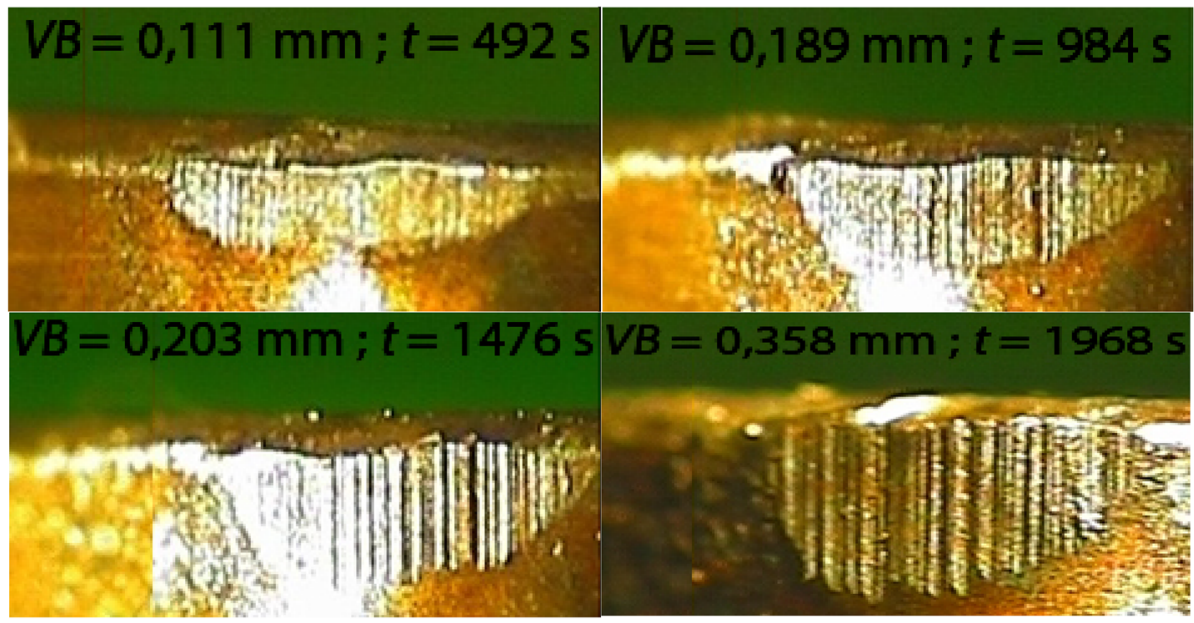

(b)

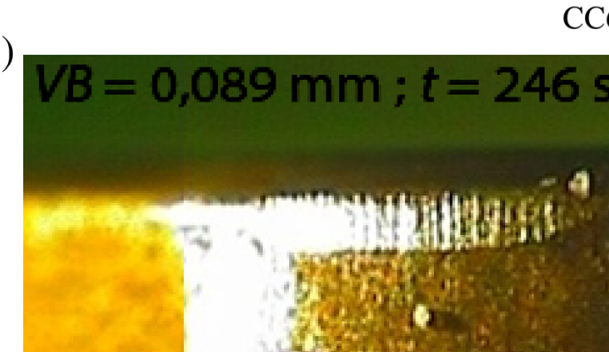

CC6050-r8
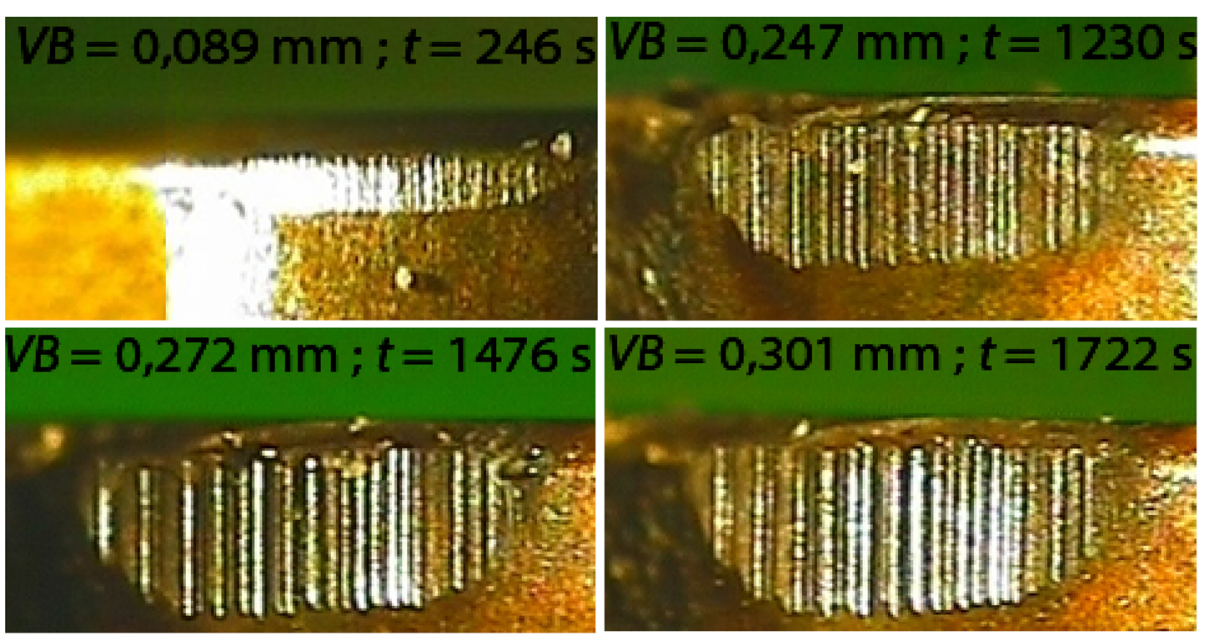

CC6050-r12

Figure 5. Flank and crater wear micrographs for CC6050 and CC650 at $V c=150 \mathrm{~m} / \mathrm{min}, f=0.08 \mathrm{~mm} / \mathrm{rev}$ and $a p=0.30 \mathrm{~mm}$. 
(c)
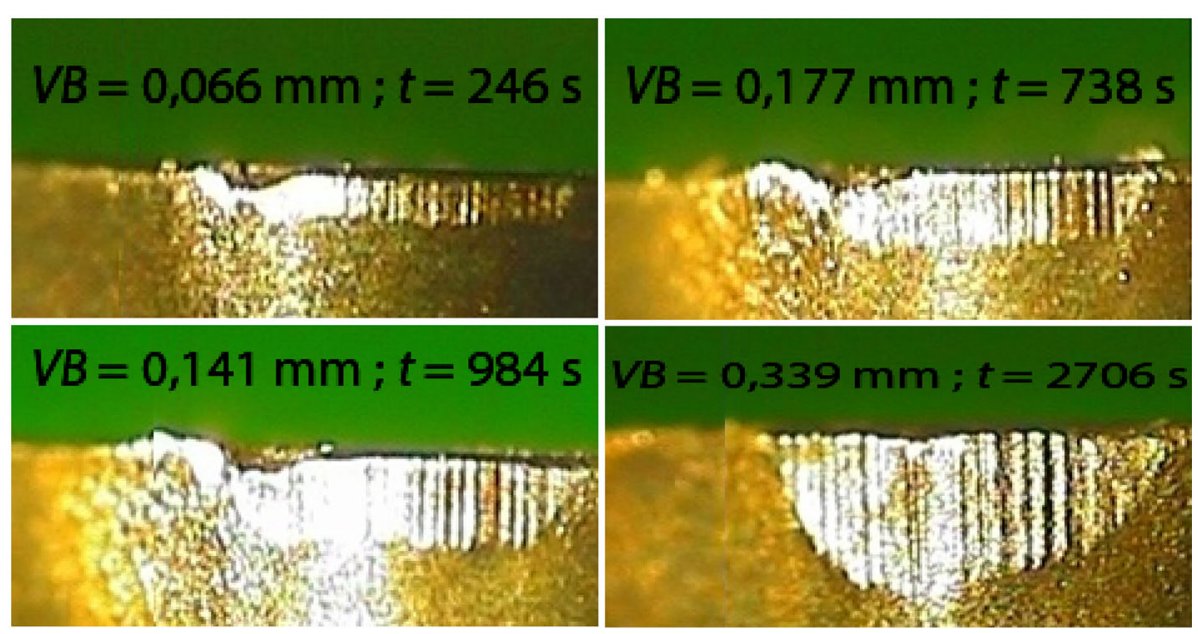

(d)

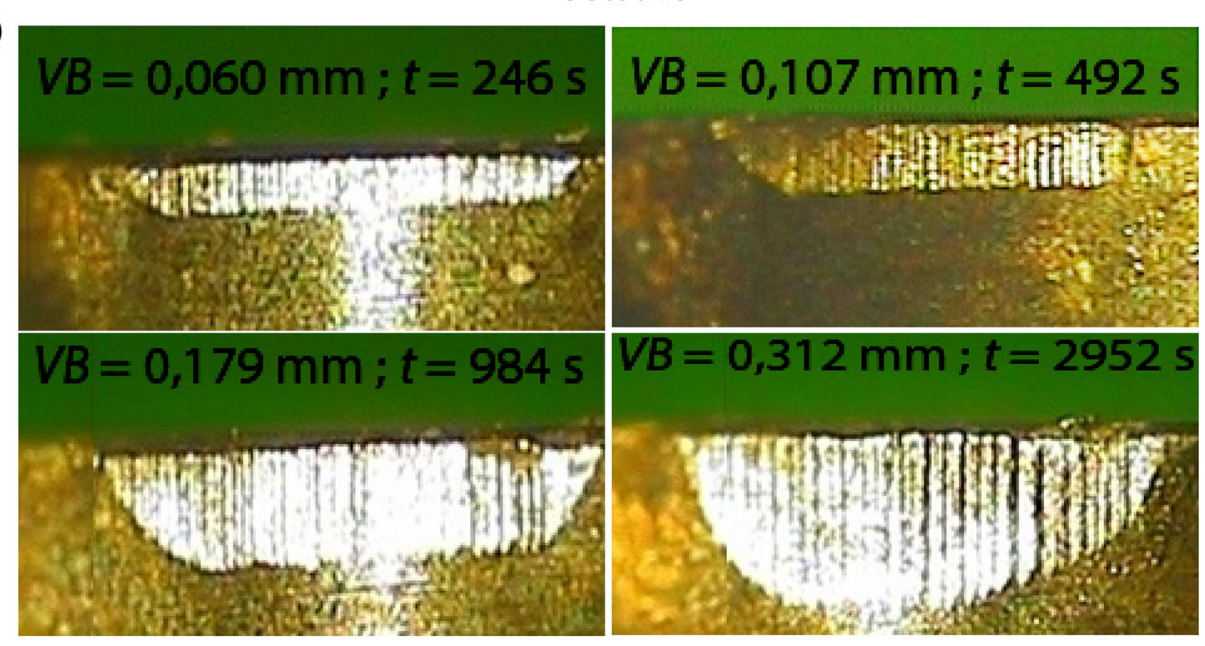

CC650-r12

Figure 5. continued

mixed ceramic inserts (CC6050 and CC650), respectively. The optimised machining forces are as follows: $\left[F a_{C C 6050}=4.4637, \quad F a_{C C 650}=5.46516 ; \quad F r_{C C 6050}-\right.$ 53.3644, $F r_{C C 650}=29.0596$ and $F t_{C C 6050}=18.7292$, $\left.F t_{C C 650}=12.3822\right] \mathrm{N}$.

\subsection{Tool life}

The tests of long duration of straight turning on AISI H11 steel treated at $50 \mathrm{HRC}$ were carried out. The purpose of these operations was to determine the wear curves as a function of machining time and, therefore, the tool life of both cutting materials used (CC6050 and CC650) at two noses radius $(0.8$ and 1.2$) \mathrm{mm}$. Figure 4 shows the evolution of the flank wear $V B$ versus machining time at $f=0.08$ $\mathrm{mm} / \mathrm{rev}, a p=0.30 \mathrm{~mm}$ and $V c=150 \mathrm{~m} / \mathrm{min}$.

Experimental observations indicate that the tool wear for both cutting tools, increased with machining time, is generally confined to three distinct regions, namely initial breakdown, uniform wear rate and rapid breakdown of the cutting edge.

According to the curve of coated ceramic CC6050 tool when cutting radius $r=0.8 \mathrm{~mm}$ and for a machining time of 4 min, the flank wear $V B$ of this insert reaches a value of $0.111 \mathrm{~mm}$. At the end of machining $t=30.50 \mathrm{~min}$, the flank wear is $0.30 \mathrm{~mm}$. This change represents an increase of $170 \%$. The tool life of this insert is $30.50 \mathrm{~min}$.

Next, for machining done by the coated ceramic CC6050 when $r=1.2 \mathrm{~mm}$, the first operation of turning by this insert leads to a value of wear $V B$ of $0.089 \mathrm{~mm}$. However, the life of the tool at the end of machining is $29 \mathrm{~min}$; the flank wear is $0.30 \mathrm{~mm}$. This change represents an increase of $237 \%$. The tool life of this insert is $29 \mathrm{~min}$.

With regard to now uncoated CC650 when cutting radius $r=0.8 \mathrm{~mm}$ and for a machining time of $4 \mathrm{~min}$, the flank wear $V B$ of this insert reaches a value of $0.066 \mathrm{~mm}$. 

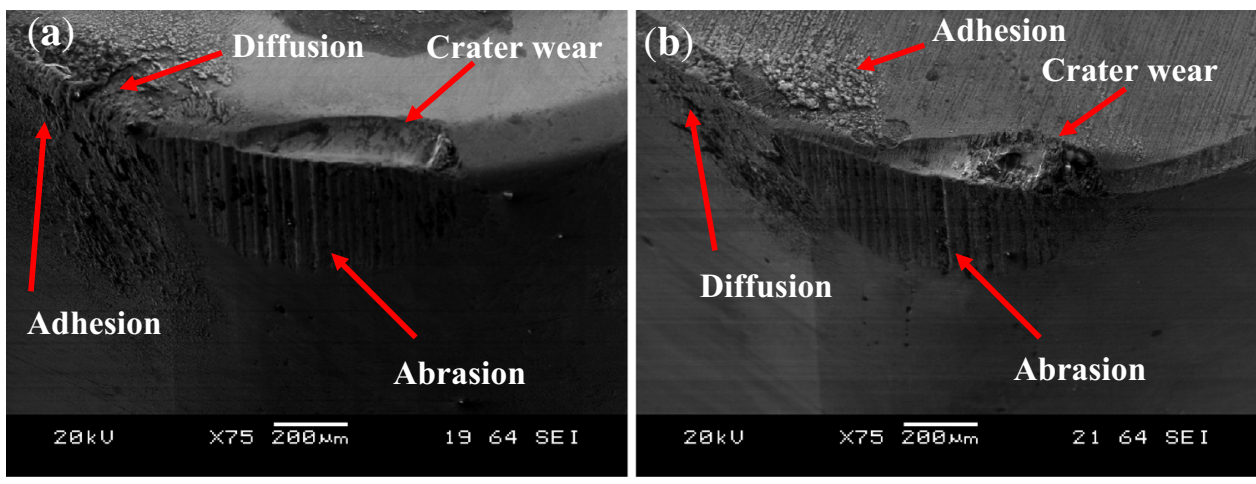

Figure 6. SEM images of the flank and crater wears of cutting tools; (a) CC6050, (b) CC650 at $V c=150 \mathrm{~m} / \mathrm{min}, f=0.08 \mathrm{~mm} / \mathrm{rev}$ and $a p=0.30 \mathrm{~mm}$ for $r=1.2 \mathrm{~min}$.

However, the life of the tool at the end of machining is $36.60 \mathrm{~min}$; the flank wear is $0.30 \mathrm{~mm}$. This change represents an increase of $334 \%$. The tool life of this insert is $36.60 \mathrm{~min}$.

Finally, for machining done by the coated ceramic CC650 when $r=1.2 \mathrm{~mm}$, the first operation of turning by this insert leads to a value of wear $V B$ of $0.060 \mathrm{~mm}$. However, the life of the tool at the end of machining is 39 min; the flank wear is $0.30 \mathrm{~mm}$. This change represents an increase of $400 \%$. The tool life of this insert is $39 \mathrm{~min}$. In general, conventional (uncoated) ceramic cutting tools CC650 for two cutting radii have a better performance compared with coated ceramic cutting tools CC6050, in particular, the tool life.

Figure $5 \mathrm{a}$ to $\mathrm{d}$ integrally illustrates the images of the rake faces, corners and secondary flank surfaces after the first 4 min of cutting time for a cutting speed of $150 \mathrm{~m} / \mathrm{min}$, feed rate $0.08 \mathrm{~mm} / \mathrm{rev}$ and depth of cut $0.30 \mathrm{~mm}$ and their final states after 39 min of straight turning on AISI H11 steel treated at $50 \mathrm{HRC}$ for coated and uncoated ceramic tools, respectively. The flank wear develops according to a regular band, which widens with cutting time for all cutting tools.

Scanning electron micrograph showing the rake and clearance faces of the ceramic cutting tools (CC6050 and CC650) after turning of AISI H11 (50 HRC) at a cutting radius of $1.2 \mathrm{~mm}$, with cutting speed, feed rate and depth of cut values of $150 \mathrm{~m} / \mathrm{min}, 0.08 \mathrm{~mm} / \mathrm{rev}$ and $0.30 \mathrm{~mm}$, respectively, are shown in figure 6 . This figure shows the typical aspect under an optical microscope of the flank wear face of ceramic tools after testing. The micrographs were taken at the end of tool life (total machining time is shown in brackets). It can be seen that abrasion, diffusion and adhesion are prominent wear mechanisms, especially for the flank and clearance faces. However, along with the nose wear, crater wear also can be seen for all the tools, indicating diffusion wear, especially for the rake face as one of the active wear mechanisms along with the abrasion and adhesion wear mechanisms. Generally, the abrasive wear has been frequently reported as a main wear mechanism in hard turning. Due to the high temperature and high stresses in hard turning, diffusion wear may also occur. Chemical reactions, including oxidation at high speeds due to high cutting temperatures, have also been reported. Chemical properties may be very important at high cutting speeds in which the cutting temperature could accelerate any chemical reaction between the tool and work piece. In conclusion, the coated TiN-mixed alumina ceramic cutting tool material is more affected by adhesive wear. Chemically activated diffusion wear is higher in TiN-mixed ceramic cutting tool materials, but the conventional CC650 ceramic tool is little affected by diffusion wear [25, 26].

As with the similarity of flank wear progress, the same phenomenon has been observed in testing the effect of the cutting time on resulting force. In order to identify that effect, testing conditions have been chosen in such a way as to permit cutting time to be greater than $32 \mathrm{~min}$ and the results are shown in figure 7 as a comparison between uncoated/coated ceramics. Figure 7 shows that cutting forces increased as a function of cutting time, and hence as a function of flank wear. This is due to wear evolution on the rake and clearance surfaces of the tool. Consequently, the work piece-tool contact surface increased together with

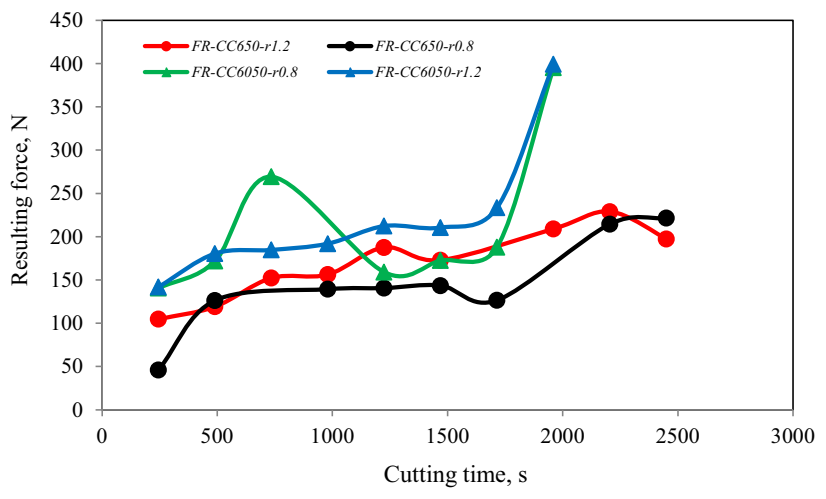

Figure 7. Influence of time on resulting force at various cutting radii for $V c=150 \mathrm{~m} / \mathrm{min}, f=0.08 \mathrm{~mm}$ and $a p=0.30 \mathrm{~mm}$ (CC6050 and CC650). 
the friction forces, generating higher resulting force. These results are similar to those mentioned by Gaitonde et al [10].

\section{Conclusion}

Based on the above results for the hard turning of AISI H11 steel with 50 HRC using coated CC6050 and uncoated CC650 ceramic under conditions similar to those used in this work, the following conclusions are made:

- Cutting force components varied almost linearly with the feed and depth of cut but showed different behaviours with cutting speed. Initially, the cutting forces decreased with the increase in cutting speed but remained almost unaltered in higher cutting speed range for both cutting ceramic tools.

- Both types of ceramic cutting tool materials undergo gradual progressive abrasive wear with increasing cutting time. Adhesive wear is higher when machining harder material. Coated TiN-mixed alumina ceramic cutting tool material is more affected by adhesive wear. Chemically activated diffusion wear is higher in TiN-mixed ceramic cutting tool materials, but conventional CC650 ceramic tool is little affected by diffusion wear.

- Experiments found that the uncoated ceramic insert (CC650) performed better than coated ceramic insert (CC6050) in terms of cutting force components ( $\mathrm{Fa}$, $F r, F t)$ and tool wear.

- Optimum values of cutting conditions are achieved with the overall desirability function. The optimum cutting conditions for cutting force components $(\mathrm{Fa}, \mathrm{Fr}$ and $F t$ ) are in the region of tool nose radius $=[0.8$ and 1.2] $\mathrm{mm}$, cutting speed $=[200$ and 100$] \mathrm{m} / \mathrm{min}$, feed rate $=0.08 \mathrm{~mm} / \mathrm{rev}$ and depth of cut $=0.10 \mathrm{~mm}$ when using uncoated mixed ceramic inserts (CC6050 and CC650), respectively.

$\begin{array}{ll}\text { List of symbols } \\ a p & \text { depth of cut, } \mathrm{mm} \\ f & \text { feed rate, } \mathrm{mm} / \mathrm{rev} \\ F a & \text { axial force, } \mathrm{N} \\ F r & \text { radial force, } \mathrm{N} \\ F t & \text { tangential force, } \mathrm{N} \\ \mathrm{HRC} & \text { Rockwell hardness } \\ r & \text { tool nose radius, } \mathrm{mm} \\ V B & \text { flank wear, mm } \\ V c & \text { cutting speed, } \mathrm{m} / \mathrm{min} \\ \alpha & \text { clearance angle, degree } \\ \gamma & \text { rake angle, degree } \\ \lambda & \text { inclination angle, degree } \\ \chi & \text { major cutting-edge angle, degree }\end{array}$

\section{References}

[1] Dureja J S, Gupat V K, Sharma V S and Dogra M 2009 Design optimization of cutting conditions and analysis of their effect on tool wear and surface roughness during hard turning of AISI-H11 steel with a coated-mixed ceramic tool. J. Eng. Manuf. 223: 1441-1450

[2] Horng J T, Liu N M and Chiang K T 2008 Investigating the machinability evaluation of Hadfield steel in the hard turning with $\mathrm{Al}_{2} \mathrm{O}_{3} / \mathrm{TiC}$ mixed ceramics tool based on the response surface methodology. J. Mater. Process. Technol. 208: $532-541$

[3] Kumar A S, Durai A J and Sornakumar T 2003 Machinability of hardened steel using alumina based ceramic cutting tools. Int. J. Ref. Met. Hard Mater. 21: 109-117

[4] Saini S, Ahuja I S and Sharma V S 2012 Influence of cutting parameters on tool wear and surface roughness in hard turning ofAISIH11 tool steel using ceramic tools. Int. J. Precis. Eng. Manuf. 13(8): 1295-1302

[5] Stachowiak G W and Stachowiak G 1994 Wear behaviour of ceramic cutting tools. Key. Eng. Mater. 96: 137-164

[6] Quiza R, Figueira, L and Davim J P 2008 Comparing statistical models and artificial neural networks on predicting the tool wear in hard machining D2 AISI steel. Int. J. Adv. Manuf. Technol. 37: 641-648

[7] Lima J G, Ávila, R F, Abrao A M, Faustino M and Davim J P 2005 Hard turning: AISI 4340 high strength alloy steel and AISI D2 cold work tool steel. J. Mater. Process. Technol. 169(3): 388-395

[8] Davim J P and Figueira L 2007 Comparative evaluation of conventional and wiper ceramic tools on cutting forces, surface roughness, and tool wear in hard turning AISI D2 steel. IMechE Part B 221: 625-633

[9] Elbah M, Yallese M A, Aouici H, Mabrouki T and Rigal J-F 2013 Comparative assessment of wiper and conventional ceramic tools on surface roughness in hard turning AISI 4140 steel. Measurement 46: 3041-3056

[10] Gaitonde V N, Karnik S R, Figueira L and Davim J P 2009 Machinability investigations in hard turning of AISI D2 cold work tool steel with conventional and wiper ceramic inserts. Int. J. Refract. Met. Hard Mater. 27: 754-763

[11] Caydas U and Hascalık A 2008 A study on surface roughness in abrasive waterjet machining process using artificial neural networks and regression analysis method. J. Mater. Process. Technol. 202: 574-582

[12] Mandal N, Doloi B and Mondal B 2010 Force prediction model of zirconia toughened alumina (ZTA) inserts in hard turning of AISI 4340 steel using response surface methodology. Int. J. Precis. Eng. Manuf. 13(9): 1589-1599

[13] Senthilkumar N, Tamizharasan T and Gobikannan S 2014 Application of response surface methodology and firefly algorithm for optimizing multiple responses in turning AISI 1045 steel. Arab. J. Sci. Eng. 39: 8015-8030

[14] Sahoo P, Barman T K and Routra B C 2008 Fractal dimension modelling of surface profile and optimisation in CNC end milling using Response Surface Method. Int. J. Manuf. Res. 3: 360-377

[15] Bouacha K, Yallese M A, Mabrouki T and Rigal J-F 2010 Statistical analysis of surface roughness and cutting forces using response surface methodology in hard turning of AISI 
52100 bearing steel with CBN tool. Int. J. Refract. Met. Hard Mater. 28: 349-361

[16] Benga G C and Abrao A M 2003 Turning of hardened 100Cr6 bearing steel with ceramic and PCBN cutting tools. J. Mater. Process. Technol. 143-144: 237-241

[17] Sahin A and Motorcu A R 2005 Surface roughness model for machining mild steel with coated carbide tool. J. Mater. Design, 26: 321-326

[18] Al-Ahmari A M A 2007 Predictive machinability models for a selected hard material in turning operations. J. Mater. Process. Technol. 190: 305-311

[19] Aouici H, Bouchelaghem H, Yallese M A, Elbah M and Fnides B 2014 Machinability investigation in hard turning of AISI D3 cold work steel with ceramic tool using response surface methodology. J. Adv. Manuf. Technol. 73: 1775-1788

[20] Meddour I, Yallese M A, Khattabi R, Elbah, M and Boulanouar L 2015 Investigation and modeling of cutting forces and surface roughness when hard turning of AISI 52100 steel with mixed ceramic tool: cutting conditions optimization. Int. J. Adv. Manuf. Technol. 77: 1387-1399

[21] Fnides B, Yallese M A, Mabrouki T and Rigal J-F 2011 Application of response surface methodology for determining cutting force model in turning hardened AISI H11 hot work tool steel. Sadhana 36(1): 109-123

[22] Aouici H, Yallese M A, Chaoui K, Mabrouki T and Rigal J-F 2012 Analysis of surface roughness and cutting force components in hard turning with CBN tool: Prediction model and cutting conditions optimization. Measurement 45: 344-353

[23] Yallese M A, Chaoui K, Zeghib N, Boulanouar L and Rigal J-F 2009 Hard machining of hardened bearing steel using cubic boron nitride tool. J. Mater. Process. Technol. 209: 1092-1104

[24] Azizi M W, Belbah A, Yallese M A, Mabrouki T and Rigal J-F 2012 Surface roughness and cutting forces modeling for optimization of machining condition in finish hard turning of AISI 52100 steel. J. Mech. Sci. Technol. 25(12): 4105-4114

[25] Arsecularatne J A, Zhang L C and Montross C 2006 Wear and tool life of tungsten carbide, PCBN, PCD cutting tools. Int. J. Mach. Tools Manuf. 46: 482-491

[26] Lahiff C, Gordon S and Phelan P 2007 PCBN tool wear modes and mechanisms in finish hard turning. Robot. Comput. Integr. Manuf. 23: 638-644 